This postprint is published in:

Proceedings of the Edinburgh Mathematical Society, Volume 63, Number 2, May 2020, 579-607

DOI: $10.1017 / S 0013091520000012$

\title{
ON STEENROD \&-HOMOLOGY, GENERALIZED MANIFOLDS, AND SURGERY
}

\author{
FRIEDRICH HEGENBARTH AND DUŠAN REPOVŠ \\ Dedicated to the memory of Professor Andrew Ranicki (1948-2018)
}

\begin{abstract}
The aim of this paper is to show the importance of the Steenrod construction of homology theories for the disassembly process in surgery on a generalized $n$-manifold $X^{n}$, in order to produce an element of generalized homology theory, which is basic for calculations. In particular, we show how to construct an element of the $n$-th Steenrod homology group $H_{n}^{s t}\left(X^{n}, \mathbb{L}^{+}\right)$, where $\mathbb{L}^{+}$is the connected covering spectrum of the periodic surgery spectrum $\mathbb{L}$, avoiding the use of the geometric splitting procedure, which is standardly used in surgery on topological manifolds.
\end{abstract}

\section{INTRODUCTION}

In order to study global objects it can be useful to decompose them into similar smaller pieces. This process of disassembly also applies to surgery theory. If one does it in an appropriate way, it produces an element of a generalized homology theory, which is basic for calculations. Here, "appropriate" means "semisimplicially" defined spectra (this holds for all spectra considered in the paper).

Geometrically, one uses transversality to attain the goal. This works well for PL topological manifolds, but it does not work for generalized manifolds. The aim of this paper is to show that for generalized manifolds an appropriate tool to overcome this problem is the Steenrod construction of homology theory.

Steenrod homology is a homology theory which is very appropriate for compact metric spaces which have certain bad local properties. Generalized Steenrod homology theory has been well presented by Ferry [5] (for more cf. also Milnor [17]). A rigorous development of this theory was given earlier by Kahn, Kaminker, and Schochet [13].

The underlying spectra of homology theory which we shall consider are $\mathbb{\Omega}^{N}, \mathbb{\Omega}^{P D}, \mathbb{\Omega}^{N P D}$, and $\mathbb{L}$. They are defined simplicially, in terms of adic

2010 Mathematics Subject Classification. Primary 55N07, 55R20, 57P10, 57R67; Secondary 18F15, 55M05, 55N20, 57P05, 57P99, 57R65.

Key words and phrases. Poincaré duality complex, generalized manifold, Steen$\operatorname{rod} \mathbb{L}$-homology, periodic surgery spectrum $\mathbb{L}$, fundamental complex, $\mathbb{L}$-homology class, Quinn index. 
objects (cf. Nicas [18], Quinn [20], and Ranicki [25]). Objects in $\mathbb{\Omega}^{N}$ (resp. $\Omega^{P D}$ ) are adic normal spaces (resp. adic Poincaré duality complexes), and objects in $\mathbb{\Omega}^{N P D}$ are adic normal spaces with boundaries being adic Poincaré duality complexes (cf. Quinn [21]).

Our main interest will be the periodic surgery spectrum $\mathbb{L}$ with

$$
\mathbb{L}_{0}=\mathbb{Z} \times{ }^{G} / T O P
$$

and its connected covering spectrum $\mathbb{L}\langle 1\rangle$, which we shall denote by $\mathbb{L}^{+}$. Elements of $\mathbb{L}^{+}$are adic surgery problems (cf. Nicas [18]), and there is a fiber sequence of spectra

$$
\mathbb{L}^{+} \rightarrow \mathbb{L} \rightarrow \mathbb{K}(\mathbb{Z}, 0)
$$

where $\mathbb{K}(\mathbb{Z}, 0)$ is the Eilenberg-MacLane spectrum.

Steenrod homology is defined on compact metric spaces $X$, and we write $H_{*}^{s t}(X, \mathcal{S})$, where $\mathcal{S}$ is any one of the above spectra. If $X$ is a PL topological manifold, then $H_{*}^{s t}(X, \mathcal{S})$ coincides with ordinarily defined generalized homology $H_{*}(X, \mathcal{S})$.

It is important to note that $\mathbb{L}^{+}$(resp. $\mathbb{L}$ ) can be defined algebraically and that the following holds.

Theorem 1.1 (Ranicki [24, 25]). There is a map of spectra

$$
\mathbb{\Omega}^{N P D} \rightarrow \Sigma \mathbb{L}^{+}
$$

where $\Sigma \mathbb{L}^{+}$is the suspension spectrum of $\mathbb{L}^{+}($cf. Ranicki [24, p.287]). Moreover, the induced morphism

$$
H_{n}\left(K, \cap^{N P D}\right) \rightarrow H_{n-1}\left(K, \mathbb{L}^{+}\right)
$$

is an isomorphism for $n \geq 4$, where $K$ is a finite polyhedron (cf. Hausmann and Vogel [8], Jones [12], Levitt [15], and Quinn [21]).

Steenrod homology is related to locally finite homology.

Theorem 1.2 (Ferry [5], Milnor [17]). For every compact metric pair $\left(X, X^{\prime}\right)$, the natural homomorphism

$$
H_{*}^{s t}\left(X, X^{\prime}, \mathcal{S}\right) \rightarrow H_{*}^{l f}\left(X \backslash X^{\prime}, \mathcal{S}\right)
$$

is an isomorphism.

We shall apply this property only for $\mathcal{S}=\mathbb{L}^{+}$. The definition of $H_{*}^{l f}\left(\cdot, \mathbb{L}^{+}\right)$ can be found in Ranicki [25, Appendix C].

In order to verify the axioms of Steenrod homology theory, one has to use the following result.

Theorem 1.3 (Ferry [5], Milnor [17]). Any compact metric pair $\left(X, X^{\prime}\right)$ can be embedded into a compact metric pair $\left(T, T^{\prime}\right)$ so that

(1) $T$ and $T^{\prime}$ are contractible;

(2) $T \backslash X$ is a $C W$-complex and $T^{\prime} \backslash X^{\prime} \subset T \backslash X$ is a subcomplex. Moreover, the construction of $\left(T, T^{\prime}\right)$ is natural with respect to maps between compact pairs $\left(X, X^{\prime}\right) \rightarrow\left(Y, Y^{\prime}\right)$. 
We shall adopt the notation from Ferry [5] and write $T \backslash X=\operatorname{OFC}(X)$ for the open fundamental complex of $X$, and $T=\operatorname{CFC}(X)$ for the closed fundamental complex of $X$. Our construction of $\operatorname{OFC}(X)$ comes with a basepoint $b_{0} \in X$. We shall describe these fundamental complexes below, because we shall construct an element in

$$
H_{n+2}^{l f}\left(\mathrm{OFC}\left(X^{n}\right) \backslash\left\{b_{0}\right\}, \cap^{N P D}\right),
$$

associated to a degree one normal map $f: M^{n} \rightarrow X^{n}$, where $X^{n}$ is a generalized $n$-manifold, and $b: \nu_{M^{n}} \rightarrow \xi$ is an appropriate bundle map.

More precisely, we have to fix a degree one normal map

$$
\left\{f_{0}, b_{0}\right\}: M_{0} \rightarrow X
$$

and associate to $\{f, b\}$ an element in

$$
H_{n+2}^{l f}\left(\mathrm{OFC}\left(X^{n}\right) \backslash\left\{b_{0}\right\}, \Omega^{N P D}\right),
$$

which we shall denote $\{f, b\}-\left\{f_{0}, b_{0}\right\}$.

By the above theorems we have the following chain of morphisms:

$$
\begin{aligned}
H_{n+2}^{l f}\left(\mathrm{OFC}\left(X^{n}\right) \backslash\left\{b_{0}\right\}, \mathbb{\Omega}^{N P D}\right) \stackrel{\cong}{\rightarrow} H_{n+2}^{s t}\left(\mathrm{CFC}\left(X^{n}\right), X^{n} \amalg\left\{b_{0}\right\}, \mathbb{\Omega}^{N P D}\right) \stackrel{\cong}{\rightarrow} \\
\stackrel{\cong}{\rightarrow} H_{n+1}^{s t}\left(\mathrm{CFC}\left(X^{n}\right), X^{n} \amalg\left\{b_{0}\right\}, \mathbb{L}^{+}\right) \rightarrow H_{n}^{s t}\left(X^{n} \amalg\left\{b_{0}\right\}, \mathbb{L}^{+}\right),
\end{aligned}
$$

i.e. $\{f, b\}-\left\{f_{0}, b_{0}\right\}$ determines an element

$$
[f, b]-\left[f_{0}, b_{0}\right] \in H_{n}^{s t}\left(X^{n} \amalg\left\{b_{0}\right\}, \mathbb{L}^{+}\right) .
$$

If $X^{n}$ is a topological $n$-manifold which carries a simplicial structure, then the construction of the element

$$
[f, b]-\left[f_{0}, b_{0}\right] \in H_{n}^{s t}\left(X^{n}, \mathbb{L}^{+}\right) \cong H_{n}\left(X^{n}, \mathbb{L}^{+}\right)
$$

follows from the splitting procedure: the surgery problem

$$
(f, b)=\left(M^{n} \stackrel{f}{\rightarrow} X^{n}, \quad \nu_{M^{n}} \stackrel{b}{\rightarrow} \xi\right)
$$

can be split into adic surgery problems which define $[f, b]$ (cf. Hegenbarth and Repovš [9] and Ranicki [25]). This is due to transversality with respect to a dual cell structure on $X^{n}$.

It is the purpose of this paper to present a construction, based on Theorems 1.1, 1.2, and 1.3, to obtain an element of $H_{n}^{s t}\left(X^{n}, \mathbb{L}^{+}\right)$which avoids this geometric splitting. We point out that algebraic splitting is also possible (cf. Pedersen, Quinn, and Ranicki [19]) and it leads to an identification of L-homology groups with controlled Wall groups.

We conclude the introduction by describing the structure of our paper. In Section 2 we shall recall preliminary material about nerves $N(\mathcal{U})$ and canonical maps

$$
\varphi: X^{n} \rightarrow N(\mathcal{U})
$$

between the underlying space $X^{n}$ and the nerve $N(\mathcal{U})$.

Section 3 will be devoted to the construction of appropriate fundamental complexes of $X^{n}$. Section 4 is the core of the paper: for any ENR Poincaré 
duality space $X^{n}$ we shall apply Theorems 1.1, 1.2, and 1.3 to construct the $\mathbb{L}$-homology class

$$
[f, b] \in H_{n}^{s t}\left(X^{n}, \mathbb{L}^{+}\right)
$$

for an arbitrary surgery problem

$$
(f, b)=\left(M^{n} \stackrel{f}{\rightarrow} X^{n}, \quad \nu_{M^{n}} \stackrel{b}{\rightarrow} \xi\right) .
$$

However, we shall see that this class depends on the canonical surgery problem (cf. Lemma 4.4).

In Section 5 we shall present some improvements and give an outlook. Finally, in Section 6 we shall discuss selected remaining related problems.

For more background information on Poincaré complexes, surgery theory, and generalized manifolds we refer the reader to e.g. [1, 2, 6, 8, 23, 26, 27, 28, 29].

\section{Coverings, nerves, AND CANONICAL MaPS}

Throughout the paper we shall consider compact metric spaces $X$. Our main interest will be closed generalized $n$-manifolds $X^{n}$, i.e. $X^{n}$ is a Euclidean neighborhood retract (ENR) and a $\mathbb{Z}$-homology $n$-manifold, i.e.

$$
H_{*}\left(X^{n}, X^{n} \backslash\{x\}\right) \cong H_{*}\left(\mathbb{R}^{n}, \mathbb{R}^{n} \backslash\{0\}\right) \text { for every } x \in X^{n}
$$

(cf. e.g. Cavicchioli, Hegenbarth, and Repovš [2]).

Open coverings $\mathcal{U}=\{U\}_{j \in J}$ will always be assumed to be locally finite. We shall denote the simplicial complex of $\mathcal{U}$ by $N(\mathcal{U})$. The vertex corresponding to $U_{j} \in \mathcal{U}$ will be denoted by $\left\langle U_{j}\right\rangle$, and if

$$
\bigcap_{0 \leq i \leq k} U_{j_{i}} \neq \emptyset
$$

then the $k$-simplex determined by $U_{j_{0}}, \ldots, U_{j_{k}} \in \mathcal{U}$ will be denoted by

$$
\sigma=\left\langle U_{j_{0}}, \ldots, U_{j_{k}}\right\rangle \in N(\mathcal{U})
$$

We shall abbreviate and write

$$
\bigcap_{0 \leq i \leq k} U_{j_{i}}=\cap \sigma .
$$

We shall also write $N(\mathcal{U})$ for its topological realization. The space $N(\mathcal{U})$ can be given the Whitehead or the metric topology. However, since we shall only consider locally finite coverings, these two topologies are identical (cf. e.g. Dugundji [3, p.99]).

\subsection{The map $\varphi: X \rightarrow N(\mathcal{U})$.}

Let

$$
\operatorname{mesh}(\mathcal{U})=\sup \{\operatorname{diam}(U) \mid U \in \mathcal{U}\}
$$

where $\operatorname{diam}(U)$ denotes the diameter of $U \subset X$. We shall now describe the first one of our canonical maps. 
A partition of unity $\left\{\varphi_{j}\right\}_{j \in J}$ subordinate to $\mathcal{U}$ gives rise to the map $\varphi$ : $X \rightarrow N(\mathcal{U})$ defined by

$$
\varphi(x)=\sum_{j} \varphi_{j}(x)\left\langle U_{j}\right\rangle .
$$

If $\left\{\bar{\varphi}_{j}\right\}_{j \in J}$ is another partition subordinate to $\mathcal{U}$, it defines the map $\bar{\varphi}: X \rightarrow$ $N(\mathcal{U})$. The homotopies

$$
\left\{t \bar{\varphi}_{j}+(1-t) \varphi_{j} \mid 0 \leq t \leq 1\right\}_{j \in J}
$$

then define a homotopy between $\bar{\varphi}$ and $\varphi$, i.e. up to homotopy, the map $\varphi$ is unique.

\subsection{Maps induced by refinements.}

Next, we shall consider refinements of coverings and induced maps. Let

$$
\mathcal{U}^{\prime}=\left\{U_{j^{\prime}}^{\prime}\right\}_{j^{\prime} \in J^{\prime}}
$$

be a refinement of $\mathcal{U}$, i.e. there is a map $s: J^{\prime} \rightarrow J$ such that

$$
U_{j^{\prime}}^{\prime} \subset U_{s\left(j^{\prime}\right)} \text { for every } j^{\prime} \in J^{\prime} .
$$

Let $\varphi^{\prime}: X \rightarrow N\left(\mathcal{U}^{\prime}\right)$ be a map as defined in Section 2.1 by the partition of unity $\left\{\varphi_{j^{\prime}}^{\prime}\right\}_{j^{\prime} \in J^{\prime}}$. We want to complete the diagram

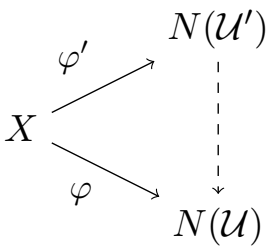

by a map indicated by the dashed line, so that it is homotopy commutative even in the controlled way.

We can get such maps from e.g. Hu [11, Theorem 8.1, p.146]: There exists a refinement $\mathcal{V}$ of $\mathcal{U}$, such that for every refinement $\mathcal{U}^{\prime}$ of $\mathcal{V}$ there is a simplicial map $N\left(\mathcal{U}^{\prime}\right) \rightarrow N(\mathcal{U})$ such that

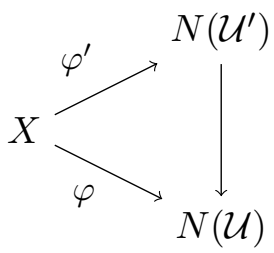

commutes up to a homotopy $h_{t}$ with

$$
\left\{h_{t}(x) \mid 0 \leq t \leq 1\right\} \subset \stackrel{\circ}{s t}\langle U\rangle \text { for some } U \in \mathcal{U} .
$$

Such maps are called bridge maps in Hu [11] and projections in Milnor [17]. 


\subsection{Maps from nerves to the space (dominations).}

We now describe the construction of maps $N(\mathcal{U}) \rightarrow X$, using the construction presented by Ferry [4, Theorems 29.7 and 29.9, Section 29]. Given

$$
\sigma=\left\langle U_{j_{0}} \ldots U_{j_{k}}\right\rangle \in N(\mathcal{U})
$$

we pick a point $x_{\sigma} \in \cap \sigma$ and define a noncontinuous map

$$
\rho: N(\mathcal{U}) \rightarrow X \text { by } \rho(\sigma)=x_{\sigma} .
$$

Let $W \subset \mathbb{R}^{m}$ be an appropriate regular neighbourhood of some embedding $X \subset \mathbb{R}^{m}$. Then the map

$$
N(\mathcal{U}) \stackrel{\rho}{\rightarrow} X \subset W
$$

can be approximated by a continuous map

$$
\psi^{\prime}: N(\mathcal{U}) \rightarrow W .
$$

The composition with the retraction

$$
\pi: W \rightarrow X
$$

then gives the map

$$
\psi=\pi \circ \psi^{\prime}: N(\mathcal{U}) \rightarrow X .
$$

By sufficiently subdividing $N(\mathcal{U})$ one can achieve that

$$
\operatorname{dist}(\psi, \rho)<\delta \text { for arbitrary small } \delta>0 \text {. }
$$

For a given $\varepsilon>0$, one then finds coverings $\mathcal{U}$ with $\operatorname{mesh}(\mathcal{U})$ sufficiently small, so that

$$
\operatorname{dist}\left(I d_{X}, \rho \circ \varphi\right)<\varepsilon
$$

and therefore

$$
\operatorname{dist}\left(I d_{X}, \psi \circ \varphi\right)<\varepsilon+\delta .
$$

By invoking Ferry [4, Corollary 29.9], we can then conclude that

$$
I d_{X} \text { and } \psi \circ \varphi \text { are } \varepsilon^{\prime} \text {-homotopic. }
$$

Beginning with an $\varepsilon^{\prime}>0$, one then finds coverings $\mathcal{U}$ of $X$ such that

$$
\psi \circ \varphi \text { is } \varepsilon^{\prime} \text {-homotopic to } I d_{X} \text {. }
$$

This is well-known (cf. e.g. Hu [11, Theorem 6.1, p.138]), but we shall need some of the details from above in the sequel.

Theorem 2.1 (Hu [11]). Let $X$ be an absolute neighborhood retract (ANR). Then the following properties hold:

(i) Given an open covering $\mathcal{U}$ of $X$, there exist maps

$$
\varphi: X \rightarrow N(\mathcal{U}) \text { and } \psi: N(\mathcal{U}) \rightarrow X .
$$

(ii) Given $\varepsilon>0$, there exists an open covering $\mathcal{U}_{\varepsilon}$ of $X$ such that the composite map

$$
X \stackrel{\varphi}{\rightarrow} N\left(\mathcal{U}_{\varepsilon}\right) \stackrel{\psi}{\rightarrow} X
$$

is $\varepsilon$-homotopic to $I d_{X}$. 
(iii) Given $\mathcal{U}_{\varepsilon}$ as in (ii), there exist a refinement $\mathcal{U}_{\varepsilon}^{\prime}$ and a map $N\left(\mathcal{U}_{\varepsilon}^{\prime}\right) \rightarrow$ $N\left(\mathcal{U}_{\varepsilon}\right)$ such that the diagram and its subdiagrams

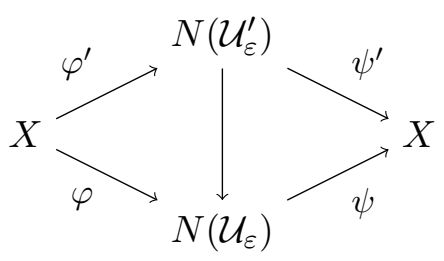

are commutative up to $\varepsilon$-homotopy.

\section{Fundamental Complexes}

Let $X$ be a compact metric space. As explained in Section 2, we can choose a covering $\mathcal{U}$ of $X$ such that the composite map

$$
X \stackrel{\varphi}{\rightarrow} N(\mathcal{U}) \stackrel{\psi}{\rightarrow} X
$$

is an $\varepsilon$-equivalence for a given $\varepsilon>0$. Then we can choose a refinement $\mathcal{U}^{\prime}$ of $\mathcal{U}$ such that the composite map

$$
X \stackrel{\varphi^{\prime}}{\rightarrow} N\left(\mathcal{U}^{\prime}\right) \stackrel{\psi^{\prime}}{\rightarrow} X
$$

is an $\varepsilon^{\prime}$-equivalence for a given $\varepsilon^{\prime}<\varepsilon$, etc.

In this way we can get a sequence of coverings $\left\{\mathcal{U}_{1}, \mathcal{U}_{2}, \ldots\right\}$ such that $\mathcal{U}_{j+1}$ refines $\mathcal{U}_{j}$ for every $j \in \mathbb{N}$, and the composite map

$$
X \stackrel{\varphi_{j}}{\rightarrow} N\left(\mathcal{U}_{j}\right) \stackrel{\psi_{j}}{\longrightarrow} X
$$

is an $\varepsilon_{j}$-equivalence with $\varepsilon_{j} \rightarrow 0$ for $j \rightarrow \infty$. Moreover, we have simplicial maps

$$
s_{j}: N\left(\mathcal{U}_{j+1}\right) \rightarrow N\left(\mathcal{U}_{j}\right)
$$

so that the diagram and its subdiagrams

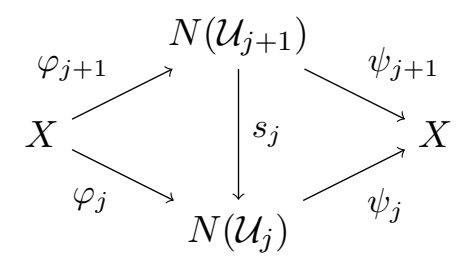

commute up to homotopy.

We add to this sequence the trivial covering $\mathcal{U}_{0}=\{X\}$ with

$$
s_{0}: N\left(\mathcal{U}_{1}\right) \rightarrow N\left(\mathcal{U}_{0}\right)=\langle X\rangle
$$

the constant map. The union of the mapping cylinders of the simplicial maps $\left\{s_{j}\right\}_{j \in\{0,1, \ldots\}}$, denoted as

$$
F=\cup_{j \geq 0} N\left(\mathcal{U}_{j+1}\right) \times I \cup_{s_{j}} N\left(\mathcal{U}_{j}\right)
$$

is an open fundamental complex of $X$. 
Let

$$
F_{l}=\underset{l \geq j \geq 0}{\cup} N\left(\mathcal{U}_{j+1}\right) \times I \cup_{s_{j}} N\left(\mathcal{U}_{j}\right),
$$

i.e. $F_{l} \subset F_{l+1}$ is a deformation retract and

$$
r_{l}: F_{l+1} \rightarrow F_{l}
$$

is the obvious retraction. Then

$$
C F=\lim _{l} F_{l}
$$

is a closed fundamental complex.

Both complexes are contractible, $F \subset C F$, and

$$
C F \backslash F=\underset{s_{j}}{\lim } N\left(\mathcal{U}_{j+1}\right) \text {. }
$$

Identifying $N\left(\mathcal{U}_{j}\right)$ with the mapping cylinder

$$
N\left(\mathcal{U}_{j+1}\right) \times I \cup \cup_{s_{j}} N\left(\mathcal{U}_{j}\right)
$$

we can form $\bigcap_{j} N\left(\mathcal{U}_{j}\right)$ and complete $F$ by it, i.e.

$$
\bigcap_{j} N\left(\mathcal{U}_{j}\right)={\underset{s_{j}}{\lim } N} N\left(\mathcal{U}_{j+1}\right)
$$

Theorem 3.1. The maps

$$
\psi_{j}: N\left(\mathcal{U}_{j}\right) \rightarrow X
$$

fit together to form the map

$$
\psi:{\underset{j}{\lim }}_{j} N\left(\mathcal{U}_{j}\right) \rightarrow X
$$

Proof. Let

$$
h: N\left(\mathcal{U}_{j+1}\right) \times I \rightarrow X
$$

be a homotopy between $\psi_{j+1}$ and $\psi_{j} \circ s_{j}$. It induces a map

$$
\Lambda_{j}: N\left(\mathcal{U}_{j+1}\right) \times I \cup_{s_{j}} N\left(\mathcal{U}_{j}\right) \rightarrow X
$$

which restricts to

$$
\psi_{j+1} \text { on } N\left(\mathcal{U}_{j+1}\right) \times\{0\} \text { and } \psi_{j} \text { on } N\left(\mathcal{U}_{j}\right),
$$

hence it can be glued to give maps

$$
F_{l}^{\circ}=\bigcup_{l \geq j \geq 1} N\left(\mathcal{U}_{j+1}\right) \times I \rightarrow X .
$$

Since the diagram

$$
N\left(\mathcal{U}_{j+1}\right) \times I \cup_{s_{j}} N\left(\mathcal{U}_{j}\right) \stackrel{r_{j}}{\longrightarrow} N\left(\mathcal{U}_{j}\right)=N\left(\mathcal{U}_{j}\right) \times\{0\}
$$

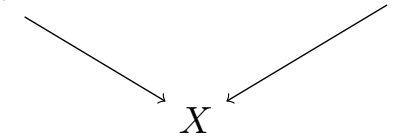


commutes, it induces a map

$$
\lim _{l \geq 1} F_{l}^{\circ} \rightarrow X
$$

whose restriction to ${\underset{l}{l \geq 1}}_{\overleftarrow{1}} N\left(\mathcal{U}_{l}\right)$ then gives the map $\psi$.

\section{Construction of $\mathbb{L}$-homology Classes}

In this section, $X^{n}$ will denote an oriented generalized $n$-manifold, $n \geq 5$, with a fundamental class

$$
[X] \in H_{n}\left(X^{n}, \mathbb{Z}\right) .
$$

Then $X^{n}$ has a Spivak normal fibration $\nu_{X^{n}}$ (cf. Quinn [22, Example 2.3]). Moreover, $\nu_{X^{n}}$ has topological reductions (cf. Ferry and Pedersen [7, Theorem 16.6]). We shall consider a sequence of coverings $\left\{\mathcal{U}_{j}\right\}_{j \in\{0,1, \ldots\}}$ as described in Section 3 .

Theorem 4.1. There is a map

$$
\Gamma_{j}: X^{n} \times I \rightarrow N\left(\mathcal{U}_{j+1}\right) \times I \cup_{s_{j}} N\left(\mathcal{U}_{j}\right)
$$

such that $\Gamma_{j}$ restricts to

$$
\varphi_{j+1}: X^{n} \times\{0\} \rightarrow N\left(\mathcal{U}_{j+1}\right) \times\{0\}
$$

and

$$
\varphi_{j}: X^{n} \times\{1\} \rightarrow N\left(\mathcal{U}_{j}\right) .
$$

Proof. We consider the composite map

$$
\bar{\varphi}_{j+1}: X^{n} \times I \stackrel{\varphi_{j+1} \times I d}{\longrightarrow} N\left(\mathcal{U}_{j+1}\right) \times I \rightarrow N\left(\mathcal{U}_{j+1}\right) \times I \sin _{j} N\left(\mathcal{U}_{j}\right) .
$$

It restricts to

$$
\varphi_{j+1}: X^{n} \times\{0\} \rightarrow N\left(\mathcal{U}_{j+1}\right) \times I \cup_{s_{j}} N\left(\mathcal{U}_{j}\right)
$$

and

$$
s_{j} \circ \varphi_{j+1}: X^{n} \times\{1\} \rightarrow N\left(\mathcal{U}_{j+1}\right) \times I \cup_{s_{j}} N\left(\mathcal{U}_{j}\right) .
$$

However,

$$
s_{j} \circ \varphi_{j+1} \simeq \varphi_{j} \text { via the homotopy } g: X^{n} \times I \rightarrow N\left(\mathcal{U}_{j}\right) .
$$

Composing $\bar{\varphi}_{j+1}$ and $g$ in the obvious way,

$$
X^{n} \times I \cup X^{n} \times[1,2] \stackrel{\bar{\varphi}_{j+1} \cup g}{\longrightarrow} N\left(\mathcal{U}_{j+1}\right) \times I \cup_{s_{j}} N\left(\mathcal{U}_{j}\right),
$$

one gets the required map

$$
\Gamma_{j}: X^{n} \times I \simeq X^{n} \times I \cup X^{n} \times[1,2] \rightarrow N\left(\mathcal{U}_{j+1}\right) \times I \cup_{s_{j}} N\left(\mathcal{U}_{j}\right) .
$$


Let us denote

$$
F_{0}=\cup_{j \geq 1} N\left(\mathcal{U}_{j+1}\right) \times I \cup_{s_{j}} N\left(\mathcal{U}_{j}\right)
$$

i.e. $F_{0} \sim F \backslash\left\{b_{0}\right\}$, where

$$
b_{0} \in N\left(\mathcal{U}_{1}\right) \times I \cup_{s_{0}} N\left(\mathcal{U}_{0}\right)
$$

is the base point of $N\left(\mathcal{U}_{0}\right)$. Then we get the following corollary.

Corollary 4.2. The maps $\Lambda_{j}$ and $\Gamma_{j}$ in Theorems 3.1 and 4.1 fit together to give maps

$$
X^{n} \times \mathbb{R}_{+} \stackrel{\Gamma}{\rightarrow} F_{0} \stackrel{\Lambda}{\rightarrow} X^{n}
$$

such that $\Lambda \circ \Gamma$ restricts to

$$
\psi_{1} \circ \varphi_{1}: X^{n} \times\{0\} \rightarrow X^{n} .
$$

We can now construct a normal space with underlying space $F_{0}$ as follows: Let $\xi$ be a topological reduction of $\nu_{X^{n}}$ and set $\eta=\Lambda^{*}(\xi)$. Since

$$
\Lambda \circ \Gamma \sim \psi_{1} \circ \varphi_{1} \sim I d
$$

we get

$$
\Gamma^{*}(\eta) \cong \xi \times \mathbb{R}_{+}
$$

Then

$$
\beta: S^{m} \times \mathbb{R}_{+} \stackrel{\alpha \times I d}{\longrightarrow} T(\xi) \times \mathbb{R}_{+} \cong T\left(\Gamma^{*}(\eta)\right) \rightarrow T(\eta)
$$

defines the structure map of the bundle $\eta$ over $F_{0}$. Here, $T($.$) denotes the$ Thom space and

$$
\alpha: S^{m} \rightarrow T(\xi) \sim T\left(\nu_{X^{n}}\right)
$$

is the structure map of $\left(X^{n}, \nu_{X^{n}}\right)$, where we assume that $X^{n} \subset S^{m}$. Therefore $\xi$ is an $\mathbb{R}^{m-n}$-bundle over $X^{n}$.

Let

$$
(f, b)=\left(f: M^{n} \rightarrow X^{n}, \quad b: \nu_{M^{n}} \rightarrow \xi\right)
$$

be a surgery problem. It defines a normal map

$$
(F, B)=\left(M^{n} \times \mathbb{R}_{+} \stackrel{f \times I d}{\longrightarrow} X^{n} \times \mathbb{R}_{+} \stackrel{\Gamma}{\rightarrow} F_{0}, \quad \nu_{M^{n}} \times \mathbb{R}_{+} \stackrel{b \times I d}{\longrightarrow} \xi \times \mathbb{R}_{+} \stackrel{\widetilde{\Gamma}}{\rightarrow} \eta\right),
$$

where $\widetilde{\Gamma}$ is the obvious bundle map covering $\Gamma$.

The mapping cylinder $M(F, B)$ is well-known to be a normal space with the boundary equal to

$$
\partial M(F, B)=M^{n} \times \mathbb{R}_{+} \amalg F_{0} .
$$

We shall only consider the restriction of $(F, B)$ to

$$
M^{n} \times(0, \infty) \rightarrow F \backslash N\left(\mathcal{U}_{1}\right) \times I \cup_{s_{0}} N\left(\mathcal{U}_{0}\right),
$$

and also denote it by $(F, B)$. Since

$$
F \backslash N\left(\mathcal{U}_{1}\right) \times I \cup_{s_{0}} N\left(\mathcal{U}_{0}\right)
$$

is a locally finite complex, normal transversality is used to decompose $M(F, B)$ into adic normal complexes. 
If $\xi^{\prime}$ is another topological reduction of $\nu_{X^{n}}$ then the same construction gives $M\left(F^{\prime}, B^{\prime}\right)$. One now glues

$$
M(F, B) \cup-M\left(F^{\prime}, B^{\prime}\right) \text { along } F_{0}
$$

to obtain an element

$$
\left\{f^{\prime}, b^{\prime}\right\}-\{f, b\} \in H_{n+2}^{l f}\left(F_{0}, \Omega^{N P D}\right) .
$$

Here, $-M(F, B)$ indicates that the orientation on $M^{n}$ is reversed.

By Theorem 1.1, this is isomorphic to

$$
H_{n+1}^{l f}\left(F_{0}, \mathbb{L}^{+}\right)
$$

which in turn, is by Theorem 1.2 , isomorphic to

$$
H_{n+1}^{s t}\left(C F, \underset{j}{\lim _{j}} N\left(\mathcal{U}_{j}\right) \amalg N\left(\mathcal{U}_{1}\right) \times I \cup_{s_{0}} N\left(\mathcal{U}_{0}\right), \mathbb{L}^{+}\right) .
$$

Under the homology boundary morphism it maps to an element in

$$
H_{n}^{s t}\left(\lim _{j} N\left(\mathcal{U}_{j}\right), \mathbb{L}^{+}\right) \text {. }
$$

Finally,

$$
\psi_{*}: H_{n}^{s t}\left(\lim _{j} N\left(\mathcal{U}_{j}\right), \mathbb{L}^{+}\right) \rightarrow H_{n}^{s t}\left(X, \mathbb{L}^{+}\right)
$$

gives the desired element $\left[f^{\prime}, b^{\prime}\right]-[f, b]$.

Remark 4.3. We thank the referee for pointing out an error here in our previous version (we have claimed that $M(F, B)$ defines an element already in $\left.H_{n+2}^{l f}\left(F_{0}, \cap^{N P D}\right)\right)$.

The element $\left\{f^{\prime}, b^{\prime}\right\}-\{f, b\}$ is represented by a compatible family of adic objects

$$
\left(\left\{f^{\prime}, b^{\prime}\right\}-\{f, b\}\right)_{\sigma}
$$

belonging to the semisimplicially defined spectrum $\mathbb{\Omega}^{N P D}$, where $\sigma$ is a simplex in $F_{0}$. Since $\sigma$ belongs to some

$$
N\left(\mathcal{U}_{l+1}\right) \times I \cup_{s_{l}} N\left(\mathcal{U}_{l}\right)
$$

one can break $\left\{f^{\prime}, b^{\prime}\right\}-\{f, b\}$ into pieces

$$
\begin{aligned}
\left\{f^{\prime}, b^{\prime}\right\}_{l}- & \{f, b\}_{l} \in H_{n+2}^{l f}\left(N\left(\mathcal{U}_{l+1}\right) \times I \cup \cup_{s_{l}} N\left(\mathcal{U}_{l}\right), \Omega^{N P D}\right)= \\
= & H_{n+2}\left(N\left(\mathcal{U}_{l+1}\right) \times I \cup_{s_{l}} N\left(\mathcal{U}_{l}\right), \Omega^{N P D}\right) .
\end{aligned}
$$

We shall return to this splitting later on. A detailed construction of the adic elements

$$
\left(\left\{f^{\prime}, b^{\prime}\right\}-\{f, b\}\right)_{\sigma}
$$

which works also in our case is given in Kühl, Macko, and Mole [14, Construction 11.3, p.236].

Before stating the main result of this section recall the following wellknown fact (cf. Ferry and Pedersen [7, Theorem 16.6]). 
Lemma 4.4. The canonical topological reduction $\xi_{0}$ of the generalized manifold $X$ defines, up to a normal cobordism, a unique surgery problem

$$
\left(f_{0}, b_{0}\right): M_{0} \rightarrow X
$$

called the canonical surgery problem.

Proof. Since $X$ is a compact metric space, it is homotopy equivalent to a finite complex $K$, hence $K$ is a $P D_{n}$-complex (cf. West [30]). There is a fiber homotopy equivalence $\nu_{K} \sim \nu_{X}$ covering the homotopy equivalence $K \sim X$. The latter induces a reduction $\xi_{0}$ on $K$ and a structure map

$$
S^{m} \rightarrow T\left(\xi_{0}\right) \text {. }
$$

Transversality applies here to define a surgery problem (the PontryaginThom construction):

$$
\left(f_{0}, b_{0}\right): M_{0} \rightarrow K \sim X
$$

If $K^{\prime}$ is another finite complex homotopy equivalent to $X$, it can be easily proved that the resulting surgery problem is normally cobordant to $\left(f_{0}, b_{0}\right)$.

In summary, we have obtained the following result.

Theorem 4.5. Let $X$ be an oriented generalized $n$-manifold, $n \geq 5$, with the canonical reduction $\xi_{0}$ of $\nu_{X}$ whose associated canonical surgery problem is

$$
\left(f_{0}, b_{0}\right): M_{0} \rightarrow X
$$

Then the procedure explained after Corollary 4.2 yields for any degree one normal map

$$
(f, b): M \rightarrow X
$$

a well-defined element

$$
[f, b]-\left[f_{0}, b_{0}\right] \in H_{n}^{s t}\left(X, \mathbb{L}^{+}\right) .
$$

\section{ImProvements AND THE OUTLOOK}

As in the previous chapter, let $X$ be a generalized manifold and $\mathcal{N}(X)$ the set of all normal bordism classes of degree one normal maps $(f, b): M \rightarrow X$. Theorem 4.5 can be improved to the following theorem.

Theorem 5.1. The association

$$
(f, b) \rightsquigarrow[f, b]-\left[f_{0}, b_{0}\right]
$$

in Theorem 4.5 defines a map

$$
t: \mathcal{N}(X) \rightarrow H_{n}^{s t}\left(X, \mathbb{L}^{+}\right) .
$$

Proof. We first have to show that the construction does not depend on the choice of the normal bordism class of $(f, b)$ and second, that it does not depend on the choice of the sequence $\left\{\mathcal{U}_{j}\right\}$ described in Chapter 3 either. 
Lemma 5.2. Fix the sequence of coverings $\left\{\mathcal{U}_{j}\right\}$ of $X$ described in Chapter 3 , Suppose that

is normally bordant to

$$
(f, b): M \rightarrow X
$$

$$
\left(f^{\prime}, b^{\prime}\right): M^{\prime} \rightarrow X
$$

Then

$$
\left\{f^{\prime}, b^{\prime}\right\}-\left\{f_{0}, b_{0}\right\}=\{f, b\}-\left\{f_{0}, b_{0}\right\} \in H_{n+2}^{l f}\left(F_{0}, \mathbb{\Omega}^{N P D}\right) .
$$

Proof. Let

$$
(g, c): W \rightarrow X \times I
$$

be a normal cobordism between $(f, b)$ and $\left(f^{\prime}, b^{\prime}\right)$. Consider also the product normal cobordism

$$
\left(g_{0}, c_{0}\right): M_{0} \times I \rightarrow X \times I .
$$

The mapping cylinders of the obvious normal maps

$$
(G, C): W \times \mathbb{R}_{+} \rightarrow X \times I \times \mathbb{R}_{+} \rightarrow F_{0} \times I
$$

and

$$
\left(G_{0}, C_{0}\right): M_{0} \times I \times \mathbb{R}_{+} \rightarrow X \times I \times \mathbb{R}_{+} \rightarrow F_{0} \times I
$$

can be glued along $F_{0} \times I$ to give a normal cobordism between

$$
M(F, B) \cup-M\left(F_{0}, B_{0}\right)
$$

and

$$
M\left(F^{\prime}, B^{\prime}\right) \cup-M\left(F_{0}, B_{0}\right),
$$

implying the claim. (For definitions of $M(F, B),-M\left(F_{0}, B_{0}\right)$, and $M\left(F^{\prime}, B^{\prime}\right)$ see the previous section.)

For the second step of the proof of Theorem 5.1, we let $\left\{\mathcal{U}_{j}\right\},\left\{\mathcal{U}_{j}^{\prime}\right\}$ be two sequences defining

$$
F_{0}=\cup_{j \geq 1} N\left(\mathcal{U}_{j+1}\right) \times I \cup_{s_{j}} N\left(\mathcal{U}_{j}\right)
$$

and

$$
F_{0}^{\prime}=\underset{j \geq 1}{\cup} N\left(\mathcal{U}_{j+1}^{\prime}\right) \times I \underset{s_{j}^{\prime}}{\cup} N\left(\mathcal{U}_{j}^{\prime}\right)
$$

Let

$$
\mathcal{U}_{j}^{\prime \prime}=\left\{U \cap U^{\prime} \mid U \in \mathcal{U}_{j}, U^{\prime} \in \mathcal{U}_{j}^{\prime}\right\}
$$

Observe that

$$
\operatorname{mesh}\left(\mathcal{U}_{j}^{\prime \prime}\right) \leq \min \left\{\operatorname{mesh}\left(\mathcal{U}_{j}\right), \operatorname{mesh}\left(\mathcal{U}_{j}^{\prime}\right)\right\}
$$

where $\left\{\mathcal{U}_{j}^{\prime \prime}\right\}$ is a sequence as described in Chapter 3 , defining $F_{0}^{\prime \prime}$ and the open (resp. closed) fundamental complex $F^{\prime \prime}$ (resp. $C F^{\prime \prime}$ ).

Let $(f, b): M \rightarrow X$ be given. Our strategy will be to compare the elements

$$
\{f, b\}-\left\{f_{0}, b_{0}\right\} \in H_{n+2}^{l f}\left(F_{0}, \Omega^{N P D}\right)
$$

and

$$
\{f, b\}^{\prime}-\left\{f_{0}, b_{0}\right\}^{\prime} \in H_{n+2}^{l f}\left(F_{0}^{\prime}, \Omega^{N P D}\right),
$$


with

$$
\{f, b\}^{\prime \prime}-\left\{f_{0}, b_{0}\right\}^{\prime \prime} \in H_{n+2}^{l f}\left(F_{0}^{\prime \prime}, \cap^{N P D}\right) .
$$

Since $\mathcal{U}_{j}^{\prime \prime}$ refines $\mathcal{U}_{j}$, there are maps

$$
p_{j}: N\left(\mathcal{U}_{j}^{\prime \prime}\right) \rightarrow N\left(\mathcal{U}_{j}\right)
$$

such that the diagram

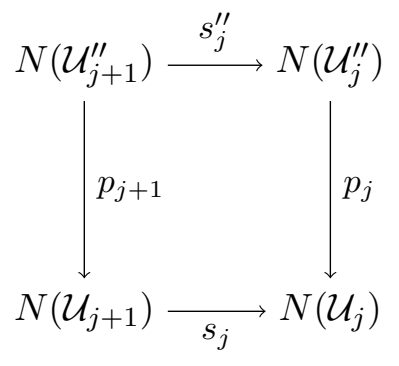

commutes up to homotopy.

The mapping cylinder construction now applies to obtain maps

$$
q_{j}: N\left(\mathcal{U}_{j+1}^{\prime \prime}\right) \times I \underset{s_{j}^{\prime \prime}}{\cup} N\left(\mathcal{U}_{j}^{\prime \prime}\right) \rightarrow N\left(\mathcal{U}_{j+1}\right) \times I \cup_{s_{j}} N\left(\mathcal{U}_{j}\right)
$$

which restrict to $p_{j+1}$ (resp. $p_{j}$ ) on the boundary. Therefore they can be pieced together to yield a map

$$
q=\cup q_{j}: F_{0}^{\prime \prime} \rightarrow F_{0} .
$$

The completion of this process then gives the map which we shall also denote by $q$,

$$
q:\left(C F^{\prime \prime}, F_{0}^{\prime \prime}, \underset{\lim _{j}}{\operatorname{li}} N\left(\mathcal{U}_{j}^{\prime \prime}\right)\right) \rightarrow\left(C F, F_{0}, \underset{\lim _{j}}{\lim } N\left(\mathcal{U}_{j}\right)\right)
$$

We shall also need the following lemma.

Lemma 5.3. Under the map

$$
q_{*}: H_{n+2}^{l f}\left(F_{0}^{\prime \prime}, \Omega^{N P D}\right) \rightarrow H_{n+2}^{l f}\left(F_{0}, \Omega^{N P D}\right),
$$

the element

maps to the element

$$
\{f, b\}^{\prime \prime}-\left\{f_{0}, b_{0}\right\}^{\prime \prime}
$$

$$
\{f, b\}-\left\{f_{0}, b_{0}\right\} .
$$

Proof. To prove the lemma, we "break up"

$$
\{f, b\}^{\prime \prime}-\left\{f_{0}, b_{0}\right\}^{\prime \prime}\left(\text { resp. }\{f, b\}-\left\{f_{0}, b_{0}\right\}\right)
$$

into pieces

$$
\{f, b\}_{j}^{\prime \prime}-\left\{f_{0}, b_{0}\right\}_{j}^{\prime \prime} \quad\left(\operatorname{resp} .\{f, b\}_{j}-\left\{f_{0}, b_{0}\right\}_{j}\right)
$$

and we show that they correspond under $q_{*}$. 
To this end, consider the normal map

$$
\left(F_{j}, B_{j}\right): M \times[j, j+1] \stackrel{(f, b) \times I d}{\longrightarrow} X \times[j, j+1] \stackrel{\Gamma_{j}}{\longrightarrow} N\left(\mathcal{U}_{j+1}\right) \times I \cup_{s_{j}} N\left(\mathcal{U}_{j}\right),
$$

where $B_{j}$ is the obvious bundle map with the target in $\Lambda_{j}^{*}(\xi)$. As above, here $b: \nu_{M} \rightarrow \xi$ is the bundle map of $(f, b): M \rightarrow X$. Observe that

$$
\left.\Lambda^{*}(\xi)\right|_{N\left(\mathcal{U}_{j+1}\right) \times I \cup N\left(\mathcal{U}_{j}\right)} \cong \Lambda_{j}^{*}(\xi) .
$$

The mapping cylinder $M\left(F_{j}, B_{j}\right)$ is then a normal complex with boundary. We do the same for

$$
\left(f_{0}, b_{0}\right): M_{0} \rightarrow X
$$

and obtain $\left(F_{j}^{\circ}, B_{j}^{\circ}\right)$.

Then

$$
M\left(F_{j}, B_{j}\right) \cup-M\left(F_{j}^{\circ}, B_{j}^{\circ}\right)
$$

defines an element

$$
\{f, b\}_{j}-\left\{f_{0}, b_{0}\right\}_{j} \in H_{n+2}\left(N\left(\mathcal{U}_{j+1}\right) \times I \cup_{s_{j}} N\left(\mathcal{U}_{j}\right), \cap^{N P D}\right) .
$$

The inclusions

$$
N\left(\mathcal{U}_{j+1}\right) \times I \cup_{s_{j}} N\left(\mathcal{U}_{j}\right) \rightarrow F_{0}
$$

represent $\{f, b\}-\left\{f_{0}, b_{0}\right\}$ as an infinite (locally finite) sum

$$
\sum_{j}\left(\{f, b\}_{j}-\left\{f_{0}, b_{0}\right\}_{j}\right) .
$$

The same process yields a representation for $\{f, b\}^{\prime \prime}-\left\{f_{0}, b_{0}\right\}^{\prime \prime}$ as an infinite (locally finite) sum

$$
\sum_{j}\left(\{f, b\}_{j}^{\prime \prime}-\left\{f_{0}, b_{0}\right\}_{j}^{\prime \prime}\right)
$$

We now consider the following (homotopy) commutative diagram

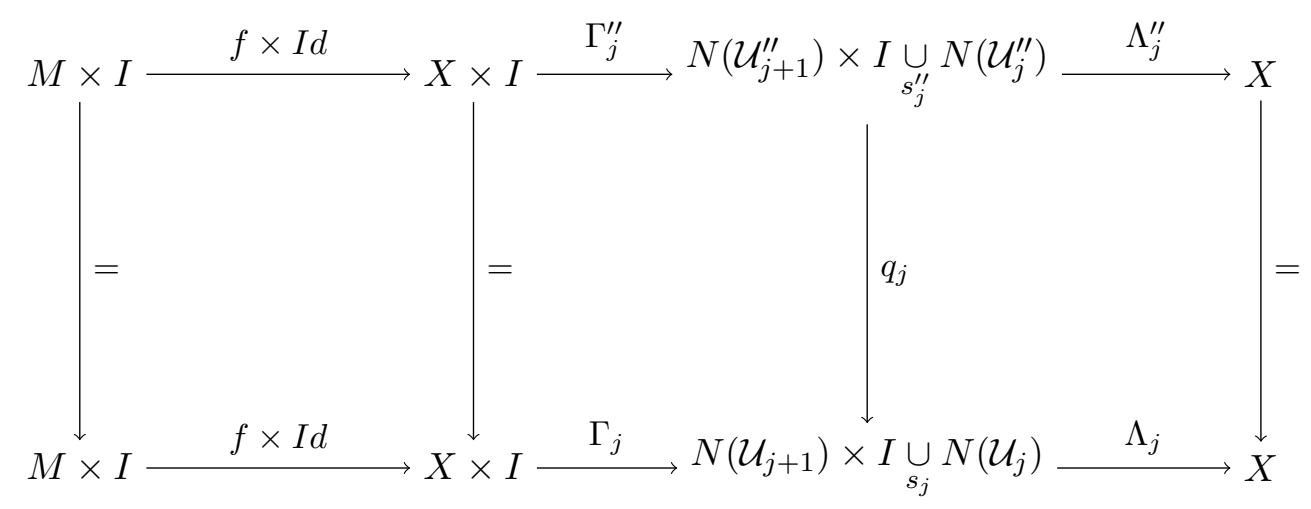


First, we deduce from this diagram that

$$
q_{j}^{*} \Lambda_{j}^{*}(\xi) \cong \Lambda_{j}^{\prime \prime *}(\xi)
$$

hence $q_{j}$ can be covered by a bundle map

$$
\bar{q}_{j}: \Lambda_{j}^{\prime \prime *}(\xi) \rightarrow \Lambda_{j}^{*}(\xi) .
$$

Next, we have the following

$$
\Gamma_{j}^{\prime \prime *}\left(\Lambda_{j}^{\prime \prime *}(\xi)\right) \cong \xi \times I \quad \text { and } \quad \Gamma_{j}^{*}\left(\Lambda_{j}^{*}(\xi)\right) \cong \xi \times I .
$$

From this we obtain the bundle maps

$$
b^{\prime \prime}: \nu_{M} \times I \stackrel{b \times I d}{\longrightarrow} \xi \times I \cong \Gamma_{j}^{\prime \prime *}\left(\Lambda_{j}^{\prime \prime *}(\xi)\right)
$$

and

$$
b: \nu_{M} \times I \stackrel{b \times I d}{\longrightarrow} \xi \times I \cong \Gamma_{j}^{*}\left(\Lambda_{j}^{*}(\xi)\right)
$$

together with the following (homotopy) commutative diagram

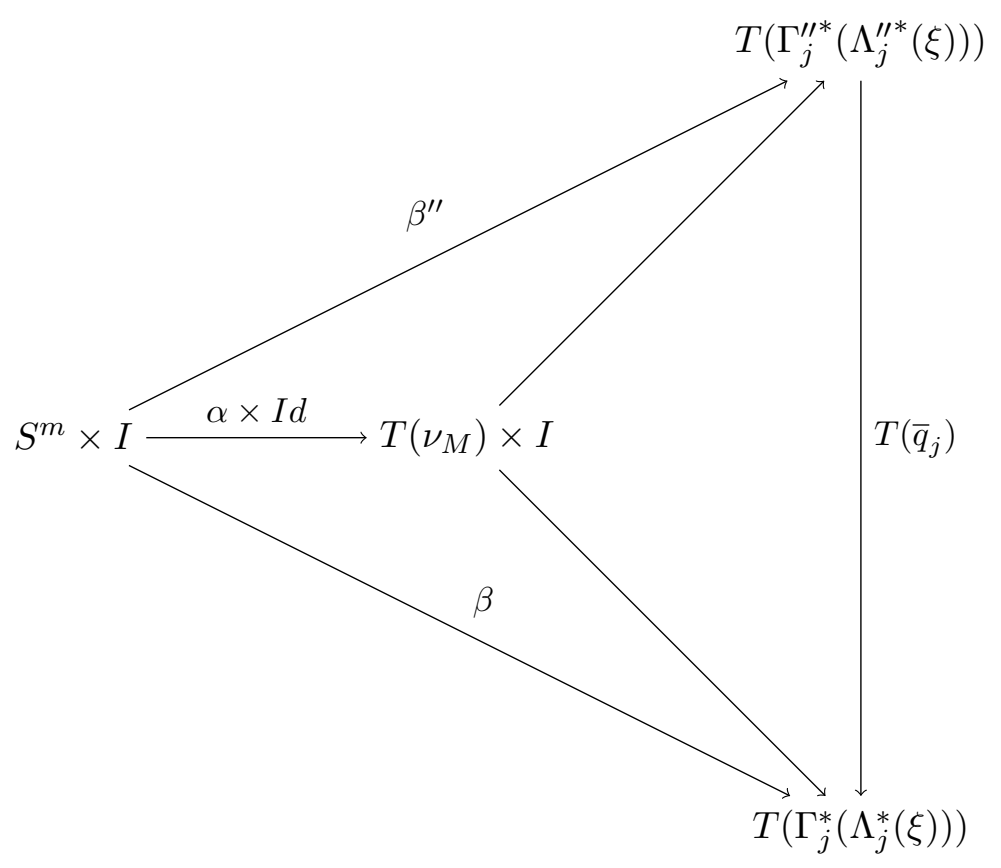

Now,

$$
\left(N\left(\mathcal{U}_{j+1}^{\prime \prime}\right) \times I{\underset{s}{\prime \prime}}_{\cup^{\prime \prime}} N\left(\mathcal{U}_{j}^{\prime \prime}\right), \Gamma_{j}^{\prime \prime *}\left(\Lambda_{j}^{\prime \prime *}(\xi)\right), \beta^{\prime \prime}\right)
$$

and

$$
\left(N\left(\mathcal{U}_{j+1}\right) \times I \cup_{s_{j}} N\left(\mathcal{U}_{j}\right), \Gamma_{j}^{*}\left(\Lambda_{j}^{*}(\xi)\right), \beta\right)
$$

determine $M\left(F_{j}^{\prime \prime}, B_{j}^{\prime \prime}\right)$ and $M\left(F_{j}, B_{j}\right)$, respectively. Therefore we can conclude that

$$
q_{j^{*}}: \Omega_{n+2}^{N P D}\left(N\left(\mathcal{U}_{j+1}^{\prime \prime}\right) \times I \underset{s_{j}^{\prime \prime}}{\cup} N\left(\mathcal{U}_{j}^{\prime \prime}\right)\right) \rightarrow \Omega_{n+2}^{N P D}\left(N\left(\mathcal{U}_{j+1}\right) \times I \cup_{s_{j}} N\left(\mathcal{U}_{j}\right)\right)
$$


maps $M\left(F_{j}^{\prime \prime}, B_{j}^{\prime \prime}\right)$ to $M\left(F_{j}, B_{j}\right)$.

The same holds for $M\left(F_{j}^{\prime \prime \circ}, B_{j}^{\prime \prime \circ}\right)$ and $M\left(F_{j}^{\circ}, B_{j}^{\circ}\right)$, if we take $\left(f_{0}, b_{0}\right)$ : $M_{0} \rightarrow X$ instead of $(f, b): M \rightarrow X$.

Since the differences have manifold boundaries, we get

$$
\begin{gathered}
M\left(F_{j}^{\prime \prime}, B_{j}^{\prime \prime}\right)-M\left(F_{j}^{\prime \prime \circ}, B_{j}^{\prime \prime \circ}\right) \in \Omega_{n+2}^{N T O P}\left(N\left(\mathcal{U}_{j+1}^{\prime \prime}\right) \times I \cup_{s_{j}^{\prime \prime}} N\left(\mathcal{U}_{j}^{\prime \prime}\right) \cong\right. \\
H_{n+2}\left(N\left(\mathcal{U}_{j+1}^{\prime \prime}\right) \times I \cup_{s_{j}^{\prime \prime}}^{\cup} N\left(\mathcal{U}_{j}^{\prime \prime}\right), \Omega^{N T O P}\right),
\end{gathered}
$$

and similarly,

$$
\begin{gathered}
M\left(F_{j}, B_{j}\right)-M\left(F_{j}^{\circ}, B_{j}^{\circ}\right) \in \Omega_{n+2}^{N T O P}\left(N\left(\mathcal{U}_{j+1}\right) \times I \cup_{s_{j}} N\left(\mathcal{U}_{j}\right)\right) \cong \\
H_{n+2}\left(N\left(\mathcal{U}_{j+1}\right) \times I \cup_{s_{j}} N\left(\mathcal{U}_{j}\right), \Omega^{N T O P}\right)
\end{gathered}
$$

(cf. Remark 5.4 below).

The canonical map of spectra $\mathbb{\Omega}^{N T O P} \rightarrow \mathbb{\Omega}^{N P D}$ maps these elements to

$$
\{f, b\}_{j}^{\prime \prime}-\left\{f_{0}, b_{0}\right\}_{j}^{\prime \prime} \text { and }\{f, b\}_{j}-\left\{f_{0}, b_{0}\right\}_{j},
$$

respectively.

The property $(*)$ above now implies that

$$
\begin{aligned}
q_{j^{*}}: & H_{n+2}\left(N\left(\mathcal{U}_{j+1}^{\prime \prime}\right) \times I \underset{s_{j}^{\prime \prime}}{\cup} N\left(\mathcal{U}_{j}^{\prime \prime}\right), \mathbb{\Omega}^{N P D}\right) \rightarrow \\
& H_{n+2}\left(N\left(\mathcal{U}_{j+1}\right) \times I \cup_{s_{j}}^{\cup} N\left(\mathcal{U}_{j}\right), \mathbb{\Omega}^{N P D}\right)
\end{aligned}
$$

$\operatorname{maps}\{f, b\}_{j}^{\prime \prime}-\left\{f_{0}, b_{0}\right\}_{j}^{\prime \prime}$ to $\{f, b\}_{j}-\left\{f_{0}, b_{0}\right\}_{j}$.

This completes the proof of the lemma.

Remark 5.4. Transversality implies that the assembly construction defines an isomorphism between $\Omega^{N T O P}$ homology groups and $\Omega^{N T O P}$ bordism groups. This is not true for $\mathbb{\Omega}^{N P D}$.

We now continue with the proof of Theorem 5.1. Denote by

$$
\langle f, b\rangle-\left\langle f_{0}, b_{0}\right\rangle \in H_{n}^{s t}\left({\underset{\lim }{j}}_{j} N\left(\mathcal{U}_{j}\right), \mathbb{L}^{+}\right)
$$

the image of $\{f, b\}-\left\{f_{0}, b_{0}\right\}$ under the composition

$$
H_{n+2}^{l f}\left(F_{0}, \Omega^{N P D}\right) \cong H_{n+1}^{s t}\left(C F, \underset{\bigsqcup_{j}}{\lim } N\left(\mathcal{U}_{j}\right) \cup\{*\}, \mathbb{L}^{+}\right) \stackrel{\partial_{*}^{\prime}}{\rightarrow} H_{n}^{s t}\left({\underset{j}{j}}_{(\lim } N\left(\mathcal{U}_{j}\right), \mathbb{L}^{+}\right),
$$

where $\partial_{*}^{\prime}$ is the composition of the boundary homomorphism with the projection

$$
H_{n}^{s t}\left({\underset{\varliminf}{j}}_{\lim _{j}} N\left(\mathcal{U}_{j}\right), \mathbb{L}^{+}\right) \oplus H_{n}^{s t}\left(\{*\}, \mathbb{L}^{+}\right) \rightarrow H_{n}^{s t}(\underbrace{\lim }_{j} N\left(\mathcal{U}_{j}\right), \mathbb{L}^{+}) .
$$

Lemma 5.3 now implies that

$$
\left(\left.q\right|_{\lim _{j}} N\left(\mathcal{U}_{j}\right)\right)_{*}\left(\langle f, b\rangle^{\prime \prime}-\left\langle f_{0}, b_{0}\right\rangle^{\prime \prime}\right)=\langle f, b\rangle-\left\langle f_{0}, b_{0}\right\rangle,
$$


where $\langle f, b\rangle^{\prime \prime}$ and $\left\langle f_{0}, b_{0}\right\rangle^{\prime \prime}$ denote the corresponding images of $\{f, b\}^{\prime \prime}$ and $\left\{f_{0}, b_{0}\right\}^{\prime \prime}$, respectively.

In order to complete the proof of Theorem 5.1, we have to pass to

$$
H_{n}^{s t}\left(X, \mathbb{L}^{+}\right)
$$

via the homomorphism

$$
\psi_{*}: H_{n}^{s t}\left({\underset{\lim }{j}}_{j} N\left(\mathcal{U}_{j}\right), \mathbb{L}^{+}\right) \rightarrow H_{n}^{s t}\left(X, \mathbb{L}^{+}\right),
$$

induced by the map

$$
\psi: \varliminf_{j}^{\lim } N\left(\mathcal{U}_{j}\right) \rightarrow X
$$

which was defined in Theorem 3.1. Similarly for the map

$$
\psi^{\prime \prime}:{\underset{j}{\lim }}_{j} N\left(\mathcal{U}_{j}^{\prime \prime}\right) \rightarrow X
$$

Now, observe that

$$
\left.q\right|_{N\left(\mathcal{U}_{j}^{\prime \prime}\right)}=p_{j}
$$

and that $\psi_{j}^{\prime \prime}$ is homotopic to $\psi_{j} \circ p_{j}$. Hence the following diagram commutes

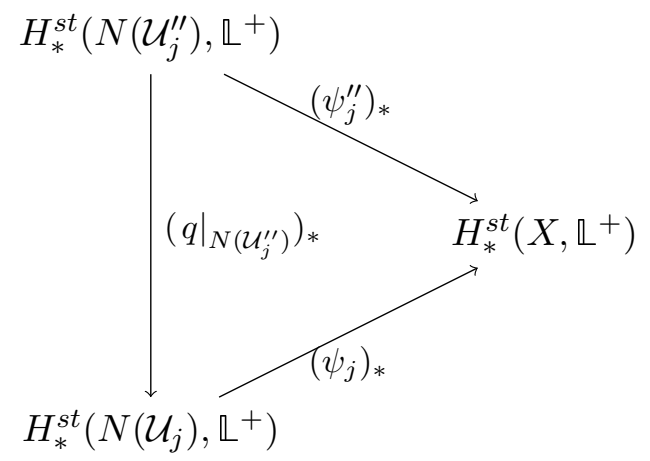

It follows that the diagram below

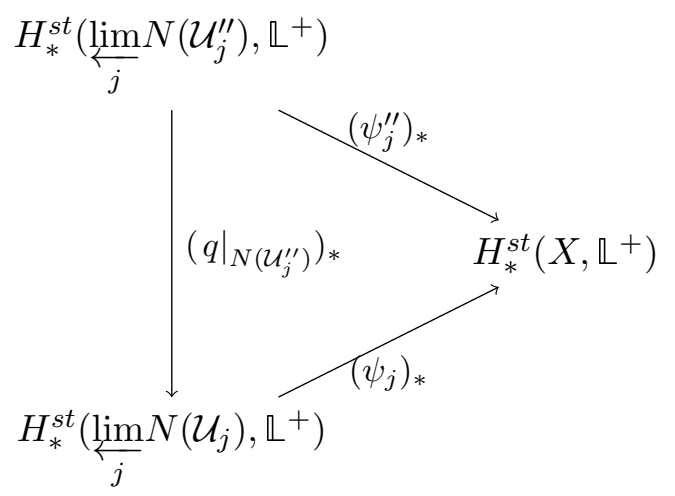


also commutes, thus we can see that

$$
\psi_{*}^{\prime \prime}\left(\langle f, b\rangle^{\prime \prime}-\left\langle f_{0}, b_{0}\right\rangle^{\prime \prime}\right)=\psi_{*}\left(\langle f, b\rangle-\left\langle f_{0}, b_{0}\right\rangle\right) .
$$

Analogously, one obtains

$$
q^{\prime}:\left(C F^{\prime \prime}, F_{0}^{\prime \prime},{\underset{j}{j}}_{\lim } N\left(\mathcal{U}_{j}^{\prime \prime}\right)\right) \rightarrow\left(C F^{\prime}, F_{0}^{\prime},{\underset{j}{\lim }}_{j} N\left(\mathcal{U}_{j}^{\prime}\right)\right)
$$

such that

$$
\psi_{*}^{\prime \prime}\left(\langle f, b\rangle^{\prime \prime}-\left\langle f_{0}, b_{0}\right\rangle^{\prime \prime}\right)=\psi_{*}^{\prime}\left(\langle f, b\rangle^{\prime}-\left\langle f_{0}, b_{0}\right\rangle^{\prime}\right) .
$$

This proves

$$
[f, b]^{\prime}-\left[f_{0}, b_{0}\right]^{\prime}=[f, b]-\left[f_{0}, b_{0}\right],
$$

and hence finally, completes the proof of Theorem 5.1 .

We shall now apply our construction to the case when $X$ is a manifold with simplicial structure. The given degree one normal map $(f, b): M \rightarrow X$ then decomposes into adic pieces to define an element

$$
\sigma_{*}^{c}(f, b) \in H_{n}\left(X, \mathbb{L}^{+}\right) .
$$

This element is the controlled surgery obstruction of $(f, b)$ over $I d: X \rightarrow X$ (cf. Pedersen, Quinn, and Ranicki [19]). We take $\left(f_{0}, b_{0}\right)=I d: X \rightarrow X$.

Supplement. In Section 4 we associated to given normal degree one maps

$$
\left(f_{0}, b_{0}\right): M_{0} \rightarrow X \quad \text { and }(f, b): M \rightarrow X,
$$

where $X$ is a generalized manifold, the element

$$
[f, b]-\left[f_{0}, b_{0}\right] \in H_{n}^{s t}\left(X, \mathbb{L}^{+}\right) .
$$

The normal maps $\left(f_{0}, b_{0}\right)$ and $(f, b)$ give rise to a normal space with boundary

$$
M_{0} \times(0, \infty) \text { and } M \times(0, \infty) .
$$

At this point, transversality for normal spaces (with TOP-manifold boundaries) is used to split (disassemble) the normal space, in order to obtain an element in the $\mathbb{\Omega}^{N P D}$-homology group.

Actually, it belongs to the $\Omega^{N T O P}$-homology, but we pass to $\Omega^{N P D}$ via

$$
\mathbb{\Omega}^{N T O P} \rightarrow \mathbb{\Omega}^{N P D} \text {. }
$$

A detailed splitting construction can be found in Kühl, Macko, and Mole [14, Construction 11.3, p.236].

If $X$ is a manifold with simplicial structure, transversality directly applies to split $(f, b)$ and $\left(f_{0}, b_{0}\right)$ into pieces in order to obtain an element in $H_{n}(X, \mathbb{L})$. It is now natural to take

$$
\left(f_{0}, b_{0}\right)=I d: X \rightarrow X .
$$

Since $I d: X \rightarrow X$ does not contribute to $\mathbb{L}$-homology, one gets an element depending on $(f, b)$ which we shall denote by

$$
\sigma(f, b) \in H_{n}(X, \mathbb{L})
$$


(this corresponds to $\operatorname{sig}_{X}^{\mathbb{L}}(f, b)$ in Kühl, Macko, and Mole [14, Definition 8.14]). Moreover,

$$
\sigma(f, b) \in H_{n}\left(X, \mathbb{\complement}^{+}\right) .
$$

Since $H_{n}(X, \mathbb{L})$ is the controlled surgery obstruction group, the element

$$
\sigma(f, b) \in H_{n}\left(X, \mathbb{L}^{+}\right) \subset H_{n}(X, \mathbb{L})
$$

is sometimes denoted by $\sigma^{c}(f, b)$.

The reason is that the 0 -dimensional components come from

$$
f^{-1}(D(\sigma, X)) \rightarrow D(\sigma, X),
$$

where $\sigma \prec X$ runs through the $n$-simplices of $X$ and $D(\sigma, X)$ is its dual with respect to a subdivision $X^{\prime}$ of $X$. Hence $D(\sigma, X)$ is a point $x \in X$ and by transversality,

$$
f^{-1}(D(\sigma, X))=\left\{ \pm y_{1}, \ldots, \pm y_{k}\right\} \subset M .
$$

Since $f$ has degree one, it is equivalent to $y \rightarrow x$, which is the trivial object. (We have also addressed such questions in Hegenbarth and Repovš [10, Lemma 2.1].)

If $X$ is only a generalized manifold, this leads to the so-called 0 -dimensional signature of $f$. This is misleading, since it is the signature obstruction of a $4 k$-dimensional surgery problem, which is "moved" to $\pi_{0}(\mathbb{L})=L_{0}$ by periodicity of $\mathbb{E}$ (cf. Hegenbarth and Repovš [10, p. 79]).

The aim of the next theorem is to show that for a given degree one normal map $(f, b): M \rightarrow X$, where $X$ is a manifold with simplicial structure, the construction via normal spaces from Section 4, gives an element which coincides with the element $\sigma(f, b)$.

Theorem 5.5. The controlled surgery obstruction of $(f, b): M \rightarrow X$ coincides with $[f, b]-\left[f_{0}, b_{0}\right]$.

Proof. Choose a sequence $\left\{\mathcal{U}_{j}\right\}$ of coverings of $X$ as above. Since $X$ is a manifold with simplicial structure, we can define

$$
\overline{\{f, b\}}-\overline{\left\{f_{0}, b_{0}\right\}} \in H_{n+2}^{l f}\left(X \times(0, \infty), \mathbb{\Omega}^{N P D}\right) .
$$

Here, $\overline{\{f, b\}}$ denotes the normal space, defined by the mapping cylinder of the map

$$
(f \times I d, b \times I d): M \times(0, \infty) \rightarrow X \times(0, \infty),
$$

and similarly, for $\overline{\left\{f_{0}, b_{0}\right\}}$.

Now, $\overline{\{f, b\}}-\overline{\left\{f_{0}, b_{0}\right\}}$ maps under the induced map

$$
\Gamma: X \times(0, \infty) \rightarrow F_{0}
$$

to

$$
\{f, b\}-\left\{f_{0}, b_{0}\right\} \in H_{n+2}^{l f}\left(F_{0}, \cap^{N P D}\right) .
$$

Under the composition

$$
H_{n+2}^{l f}\left(X \times(0, \infty), \mathbb{\Omega}^{N P D}\right) \cong H_{n+1}^{l f}\left(X \times(0, \infty), \mathbb{L}^{+}\right) \cong
$$




$$
H_{n+1}^{s t}\left(X \times[0, \infty] /(X \times\{\infty\}), X \cup\{*\}, \mathbb{L}^{+}\right) \stackrel{\partial_{*}^{\prime}}{\rightarrow} H_{n}^{s t}\left(X, \mathbb{L}^{+}\right),
$$

$\overline{\{f, b\}}-\overline{\left\{f_{0}, b_{0}\right\}}$ maps to $\sigma_{*}^{c}(f, b)$. This is because $\overline{\{f, b\}}-\overline{\left\{f_{0}, b_{0}\right\}}$ is represented by the mapping cylinders of

$$
(f \times I d, b \times I d) \text { and }\left(f_{0} \times I d, b_{0} \times I d\right) .
$$

The latter one does not contribute to $\mathbb{L}$-homology because we have chosen $\left(f_{0}, b_{0}\right)=(I d, I d)$. Under the composition it therefore goes to the element defined by splitting $(f, b): M \rightarrow X$, i.e. to $\sigma(f, b)$.

Here,

$$
X \times[0, \infty] /(X \times\{\infty\})
$$

is the completion of $X \times(0, \infty)$ obtained as the inverse limit, similarly as

$$
C F=\underset{l}{\lim } F_{l}
$$

(cf. Chapter 3). However, under

$$
\Gamma_{*}: H_{n+2}^{l f}\left(X \times(0, \infty), \Omega^{N P D}\right) \rightarrow H_{n+2}^{l f}\left(F_{0}, \Omega^{N P D}\right)
$$

the difference $\overline{\{f, b\}}-\overline{\left\{f_{0}, b_{0}\right\}}$ maps to $\{f, b\}-\left\{f_{0}, b_{0}\right\}$.

Consider now (using previous notations)

$$
X \times I \stackrel{\Gamma_{j}}{\longrightarrow} N\left(\mathcal{U}_{j+1}\right) \times I \underset{s_{j}}{\cup} N\left(\mathcal{U}_{j}\right) \stackrel{\bar{\Lambda}_{j}}{\longrightarrow} X \times I,
$$

where

$$
\bar{\Lambda}_{j}(u, t)=\left(\Lambda_{j}(u), t\right)
$$

So

$$
\bar{\Lambda}_{j} \circ \Gamma_{j}(x, 0)=\left(\left(\psi_{j+1} \circ \varphi_{j+1}\right)(x), 0\right)
$$

and

$$
\bar{\Lambda}_{j} \circ \Gamma_{j}(x, 1)=\left(\left(\psi_{j} \circ \varphi_{j}\right)(x), 1\right) .
$$

Since $\psi_{k} \circ \varphi_{k} \sim I d_{X}$ we can use these homotopies to glue the maps and obtain

$$
X \times \mathbb{R}_{+} \stackrel{\Gamma}{\rightarrow} F_{0} \stackrel{\bar{\Lambda}}{\rightarrow} X \times \mathbb{R}_{+},
$$

restricting to

$$
X \times\{0\} \stackrel{\varphi}{\rightarrow}{\underset{\lim }{j}}_{j} N\left(\mathcal{U}_{j}\right) \stackrel{\psi}{\rightarrow} X \times\{0\},
$$

i.e. we get maps

$$
X \times[0, \infty] /(X \times\{\infty\}) \rightarrow C F \rightarrow X \times[0, \infty] /(X \times\{\infty\}) .
$$

Therefore $\Gamma$ induces a morphism between the sequences

$$
\begin{gathered}
H_{n+2}^{l f}\left(X \times[0, \infty), \mathbb{Q}^{N P D}\right) \cong H_{n+1}^{l f}\left(X \times[0, \infty), \mathbb{L}^{+}\right) \cong \\
H_{n+1}^{s t}\left(X \times[0, \infty] /(X \times\{\infty\}), X \cup\{*\}, \mathbb{L}^{+}\right) \stackrel{\partial_{*}^{\prime}}{\longrightarrow} H_{n}^{s t}\left(X, \mathbb{L}^{+}\right),
\end{gathered}
$$

and

$$
H_{n+2}^{l f}\left(F_{0}, \cap^{N P D}\right) \cong H_{n+1}^{l f}\left(F_{0}, \mathbb{L}^{+}\right) \cong
$$




$$
\left.H_{n+1}^{s t}\left(C F,{\underset{j}{j}}_{\lim } N\left(\mathcal{U}_{j}\right) \cup\{*\}, \mathbb{L}^{+}\right) \stackrel{\partial_{*}^{\prime}}{\longrightarrow} H_{n}^{s t}{\underset{亡}{\lim } N}_{\lim _{j}}\left(\mathcal{U}_{j}\right), \mathbb{L}^{+}\right) .
$$

It follows that

$$
\varphi_{*}\left(\sigma_{*}^{c}(f, b)\right)=\langle f, b\rangle-\left\langle f_{0}, b_{0}\right\rangle \in H_{n}^{s t}\left({\underset{\leftarrow}{j}}_{\lim _{j}} N\left(\mathcal{U}_{j}\right), \mathbb{L}^{+}\right) .
$$

Since $\psi \circ \varphi \sim I d$, we can conclude that

$$
[f, b]-\left[f_{0}, b_{0}\right]=\psi_{*}\left(\langle f, b\rangle-\left\langle f_{0}, b_{0}\right\rangle\right)=\psi_{*}\left(\varphi_{*}\left(\sigma_{*}^{c}(f, b)\right)\right)=\sigma_{*}^{c}(f, b) .
$$

We shall finish this section with some remarks on the map $t$. In the PL manifold case there is an $\mathbb{L}^{\bullet}$-orientation

$$
\mathcal{U}_{\mathbb{L}} \bullet \in \bar{H}^{m-n}\left(T\left(\nu_{X}\right), \mathbb{L} \bullet\right),
$$

where $\mathbb{L}^{\bullet}$ is the symmetric $\mathbb{L}$-spectrum. Furthermore, $\mathbb{L}^{\bullet}$ is a ring spectrum and $\mathbb{L}^{+}$is an $\mathbb{L}^{\bullet}$-module spectrum, and the cup product

$$
. \cup \mathcal{U}_{\mathbb{L}} \bullet:[X, G / T O P]=H^{\circ}\left(X, \mathbb{L}^{+}\right) \rightarrow \bar{H}^{m-n}\left(T\left(\nu_{X}\right), \mathbb{L}^{+}\right)
$$

is an isomorphism. Here we are assuming that $X \subset \mathbb{R}^{m}$.

The difference between $(f, b): M \rightarrow X$ and $\left(f_{0}, b_{0}\right): X \rightarrow X$ defines a map

$$
\mathcal{N}(X) \rightarrow[X, G / T O P]
$$

Combining with the Alexander-Spanier duality

$$
\bar{H}^{m-n}\left(T\left(\nu_{X}\right), \mathbb{L}^{+}\right) \cong H_{n}\left(X, \mathbb{L}^{+}\right),
$$

we obtain a bijective map

$$
\mathcal{N}(X) \rightarrow H_{n}\left(X, \mathbb{L}^{+}\right) .
$$

This is the map $t$ (cf. Ranicki [25, Chapter 17, pp.191-193]).

In the case of a generalized manifold we can embed $X$ into $\mathbb{R}^{m}$ with a cylindrical neighborhood, also obtaining an isomorphism

$$
\bar{H}^{m-n}\left(T\left(\nu_{X}\right), \mathbb{L}^{+}\right) \cong H_{n}^{s t}\left(X, \mathbb{L}^{+}\right) .
$$

Let

$$
N=\partial N \times I \cup_{p} X
$$

be a mapping cylinder neighborhood of $X \subset S^{m+1}$. It can be used to prove the following fact.

Theorem 5.6. There exists an $\mathbb{\complement} \bullet$-orientation

$$
\mathcal{U} \in H^{m+1-n}(N, \partial N, \mathbb{L} \bullet) .
$$

and an isomorphism

$$
. \cup \mathcal{U}: H^{0}\left(X, \mathbb{L}^{+}\right) \stackrel{\cong}{\rightarrow} H^{m+1-n}\left(N, \partial N, \mathbb{L}^{+}\right) .
$$


With this theorem one obtains the following isomorphisms:

$$
\begin{gathered}
H^{0}\left(X, \mathbb{L}^{+}\right) \cong H^{m+1-n}\left(N, \partial N, \mathbb{L}^{+}\right) \cong H^{m+1-n}\left(S^{m+1}, S^{m+1} \backslash N, \mathbb{L}^{+}\right) \cong \\
H^{m+1-n}\left(S^{m+1}, S^{m+1} \backslash X, \mathbb{L}^{+}\right) \cong \\
\bar{H}^{m-n}\left(S^{m+1} \backslash X, \mathbb{L}^{+}\right) \cong H_{n}^{s t}\left(X, \mathbb{L}^{+}\right) .
\end{gathered}
$$

The last isomorphism is the Steenrod duality (cf. Kahn, Kaminker and Schochet [13, Theorem B], there one must take the reduced $\mathbb{L}^{+}$-homology).

We shall omit the proof of Theorem 5.6 because it is not obvious that the composition

$$
\mathcal{N}(X) \rightarrow H^{0}\left(X, \mathbb{L}^{+}\right) \rightarrow H_{n}^{s t}\left(X, \mathbb{L}^{+}\right)
$$

coincides with the association

$$
(f, b) \rightarrow[f, b]-\left[f_{0}, b_{0}\right]
$$

This will be included in our future paper.

\section{Discussion}

(I). The homotopy groups of the spectrum $\mathbb{L}^{+}$are the Wall groups of the trivial group, i.e.

$$
\pi_{n}\left(\mathbb{L}^{+}\right) \cong L_{n}(1) \cong L_{n} \text { for every } n \geq 1
$$

Since the simplicial complex

$$
N\left(\mathcal{U}_{1}\right) \times I \cup \cup_{s_{0}} N\left(\mathcal{U}_{0}\right)
$$

is contractible, we have

$$
\begin{gathered}
H_{n}^{s t}\left(N\left(\mathcal{U}_{1}\right) \times I \cup_{s} N\left(\mathcal{U}_{0}\right), \mathbb{L}^{+}\right) \cong H_{n}\left(N\left(\mathcal{U}_{1}\right) \times I \cup_{s_{0}} N\left(\mathcal{U}_{0}\right), \mathbb{L}^{+}\right) \cong \\
\cong H_{n}\left(\left\{b_{0}\right\}, \mathbb{L}^{+}\right) \cong L_{n} .
\end{gathered}
$$

Therefore the above mentioned homology boundary homomorphism is

$$
H_{n+1}^{s t}\left(C F,{\underset{j}{j}}_{\lim _{j}} N\left(\mathcal{U}_{j}\right) \amalg\left\{b_{0}\right\}, \mathbb{L}^{+}\right) \rightarrow H_{n}^{s t}\left({\underset{\leftarrow}{j}}_{\lim } N\left(\mathcal{U}_{j}\right), \mathbb{L}^{+}\right) \oplus L_{n} .
$$

The component in $L_{n}$ is the surgery obstruction of

$$
(f, b)=\left(M^{n} \rightarrow X^{n}, \quad \nu_{M^{n}} \rightarrow \xi\right)
$$

mapped to $L_{n}$ under

$$
L_{n}\left(\pi_{1}\left(X^{n}\right)\right) \rightarrow L_{n}(1) \cong L_{n}
$$

where the morphism is induced by $X^{n} \rightarrow\{*\}$. 
(II). We have used the map

$$
\psi_{*}: H_{n}^{s t}\left({\underset{\lim }{\lim }} N\left(\mathcal{U}_{j}\right), \mathbb{L}^{+}\right) \rightarrow H_{n}^{s t}\left(X^{n}, \mathbb{L}^{+}\right)
$$

to obtain our element

$$
[f, b] \in H_{n}^{s t}\left(X^{n}, \mathbb{L}^{+}\right) .
$$

We did not need that it is an isomorphism.

In fact, the relation between $\underset{j}{\lim } N\left(\mathcal{U}_{j}\right)$ and $X^{n}$ seems to be insufficiently documented. It was claimed in Milnor [17, Lemma 2] that they are identical. It was also asserted in Ferry [5, Footnote, p. 156] that they are strongly shape equivalent.

To this end, we state the following theorem. First, recall that given $\varepsilon>0$, a map $f: X \rightarrow Y$ of metric spaces $X$ and $Y$ is called an $\varepsilon$-map if for every $y \in Y, \operatorname{diam}\left(f^{-1}(y)\right)<\varepsilon$.

Theorem 6.1. The maps

$$
\varphi_{j}: X^{n} \rightarrow N\left(\mathcal{U}_{j+1}\right) \times I \cup_{s_{j}} N\left(\mathcal{U}_{j}\right)
$$

fit together to produce the map

$$
\varphi: X^{n} \rightarrow \underset{\lim _{j}}{ } N\left(\mathcal{U}_{j}\right)
$$

which is an $\varepsilon$-map onto the image of $\varphi$ for all $\varepsilon>0$.

Proof. The maps $\Gamma_{j}$ can be glued to get maps

$$
X_{l}^{n}=X^{n} \times[0, l+1] \rightarrow F_{l}^{\circ},
$$

such that the diagram

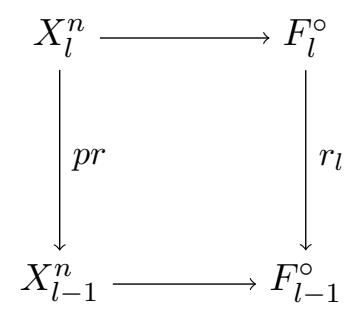

commutes.

Hence we get a map

$$
X^{n} \times[0, \infty] \rightarrow \underset{l}{\lim } F_{l}^{\circ}
$$

which restricts to

$$
\varphi: X^{n} \times\{\infty\} \rightarrow \underset{j}{\lim _{j}} N\left(\mathcal{U}_{j}\right)
$$

If now

$$
p_{l}: \varliminf_{j} \lim _{j} N\left(\mathcal{U}_{j}\right) \rightarrow N\left(\mathcal{U}_{l}\right)
$$


is the projection, then $p_{l} \circ \varphi=\varphi_{l}$.

Let $x \in \operatorname{Im} \varphi$. Then

$$
x_{l}=p_{l}(x) \in N\left(\mathcal{U}_{l}\right)
$$

belongs to some st $(\langle U\rangle)$, for some vertex $\langle U\rangle \in N\left(\mathcal{U}_{l}\right)$, where $U \in \mathcal{U}_{l}$.

Therefore

$$
\varphi^{-1}(x) \subset \varphi_{l}^{-1}\left(x_{l}\right) \subset U
$$

(cf. Dugundji [3, Theorem 5.4, Chapter VIII]). Hence

$$
\operatorname{diam}\left(\varphi^{-1}(x)\right) \leq \operatorname{mesh}\left(\mathcal{U}_{l}\right)
$$

and since $\operatorname{mesh}\left(\mathcal{U}_{j}\right) \rightarrow 0$ for $j \rightarrow \infty$, the assertion follows.

Remark 6.2. It would be interesting to know if the Bing Shrinking Criterion (cf. Marin and Visetti [16]) can be applied to improve Theorem 6.1.

\section{ACKNOWLEDGEMENTS}

This research was supported by the Slovenian Research Agency grants P1-0292, J1-8131, N1-0064, N1-0083, and N1-0114. We very gratefully acknowledge the referee for several important comments and suggestions which have considerably improved the presentation.

\section{REFERENCES}

[1] J.L. Bryant, S. Ferry, W. Mio, and S. Weinberger, Topology of homology manifolds, Ann. of Math. 143 (2) (1996), 435-467. MR 97b:57017

[2] A. Cavicchioli, F. Hegenbarth, and D. Repovš, Higher-Dimensional Generalized Manifolds: Surgery and Constructions, EMS Series of Lectures in Mathematics 23, European Mathematical Society, Zürich, 2016. MR 3558558

[3] J. Dugundji, Topology, Allyn and Bacon, Boston, 1973. MR 33\#1824

[4] S.C. Ferry, Geometric Topology Notes, Unpubl. manuscr., Rutgers Univ., Piscataway, NJ, 2008. https://sites.math.rutgers.edu/ sferry/ps/geotop.pdf

[5] S.C. Ferry, Remarks on Steenrod homology, Novikov Conjectures, Index Theorems, and Rigidity, Vol. 2, S. Ferry, A. Ranicki, and J. Rosenberg, Eds., London Math. Soc. Lecture Note Ser. 227, Cambridge University Press, Cambridge, 1995, pp. 148-166. MR 98d:55004

[6] S.C. Ferry, Epsilon-delta surgery over $\mathbb{Z}$, Geom. Dedicata 148 (2010), 71-101. MR 2011m:57030

[7] S.C. Ferry and E.K. Pedersen, Epsilon surgery theory, Novikov Conjecture, Index Theorem and Rigidity, Vol. 2, S. Ferry, A. Ranicki, and J. Rosenberg, Eds., London Math. Soc., Lecture Notes Series 227, London, 1995, pp. 167-226. MR 97g:57044

[8] J.C. Hausmann and P. Vogel, Geometry on Poincaré Spaces, Math. Notes 41, Princeton University Press, Princeton, NJ, 1993. MR 95a:57042

[9] F. Hegenbarth and D. Repovš, Controlled homotopy equivalences on structure sets of manifolds, Proc. Amer. Math. Soc. 142 (2014), 3987-3999. MR 3251739

[10] F. Hegenbarth and D. Repovš, Controlled surgery and [-homology. Mediterr. J. Math. 16 (2019), no. 3, Art. 79, 22 pp. MR 3945264

[11] S.T. Hu, Theory of Retracts, Wayne State Univ. Press, Detroit, 1965. MR 31\#6202

[12] L. Jones, Patch spaces: a geometric representation for Poincaré spaces, Ann. of Math. (2) 97 (1973), 306-343. MR 0315720; Corrections, Ann. of Math. (2) 102 (1975), 183-185. MR 0391108 
[13] D.S. Kahn, J. Kaminker, and C. Schochet, Generalized homology theories on compact metric spaces, Michigan Math. J. 24 (2) (1977), 203-224. MR 0474274

[14] P. Kühl, T. Macko, and A. Mole, The total surgery obstruction revisited, Münster J. Math. 6 (2013), 181-269. MR3148212

[15] N. Levitt, Poincaré duality cobordism, Ann. of Math. (2) 96 (1972), 211-244. MR 0314059

[16] A. Marin and Y.M. Visetti, A general proof of Bing's shrinkability criterion, Proc. Amer. Math. Soc. 53 (2) (1975), 501-507. MR 52\#9156

[17] J. Milnor, On the Steenrod homology theory, Novikov Conjectures, Index Theorems, and Rigidity, Vol. 1, S. Ferry, A. Ranicki, and J. Rosenberg, Eds., London Math. Soc. Lecture Note Ser. 226, Cambridge University Press, Cambridge, 1995, pp. 7996. MR 98d:55005

[18] A. Nicas, Induction theorems for groups of homotopy manifold structures, Mem. Amer. Math. Soc. 39 (267), Amer. Math. Soc., Providence, RI, 1982. MR 83i:57026

[19] E.K. Pedersen, F. Quinn, and A. Ranicki, Controlled surgery with trivial local fundamental groups, High-Dimensional Manifold Topology, F.T. Farrell and W. Lück, Eds., World Sci. Publishing, River Edge, N.J., 2003, pp. 421-426. MR 2005e:57077

[20] F.S. Quinn, A Geometric Formulation of Surgery, Doctoral Dissertation, Princeton University, Princeton, 1969. MR 2619602

[21] F.S. Quinn, Surgery on Poincaré and normal spaces, Bull. Amer. Math. Soc. 78 (1972), 262-267. MR 0296955

[22] F.S. Quinn, Resolutions of homology manifolds and the topological characterization of manifolds, Invent. Math. 72 (2) (1983), 267-284; Corrigendum, Invent. Math. 85 (3) (1986), 653. MR 85b:57023, MR 87g:57031

[23] F.S. Quinn, An obstruction to the resolution of homology manifolds, Michigan Math. J. 34 (2) (1987), 285-291. MR 88j:57016

[24] A.A. Ranicki, The total surgery obstruction, Proc. Alg. Topol. Conf. Aarhus 1978, Lect. Notes Math., Springer-Verlag, Berlin 763 (1979), pp. 275-316. MR 81e:57034

[25] A.A. Ranicki, Algebraic L-Theory and Topological Manifolds, Cambridge Tracts in Math. 102, Cambridge Univ. Press, Cambridge, 1992. MR 94i:57051

[26] M. Yamasaki, L-groups of crystallographic groups, Invent. Math. 88 (3) (1987), 571-602. MR 88c:57017

[27] C.T.C. Wall, Poincaré complexes, I. Ann. of Math. 86 (2) (1967), 213-245. MR $36 \# 880$

[28] C.T.C. Wall, Surgery on Compact Manifolds, Second Ed., Edited and with a foreword by A.A. Ranicki, Mathematical Surveys and Monographs 69, American Mathematical Society, Providence, RI, 1999. MR1687388

[29] S. Weinberger, Homology manifolds, Handbook of Geometric Topology, NorthHolland, Amsterdam, 2002, 1085-1102. MR 2003b:57032

[30] J.E. West, Mapping Hilbert cube manifolds to ANR's: A solution of a conjecture of Borsuk, Ann. of Math. (2) 106 (1977), 1-18. MR 56 \#9534

Dipartimento di Matematica "Federigo Enriques", Università degli studi Di Milano, 20133 Milano, Italy

Email address: friedrich.hegenbarth@unimi.it

Faculty of Education and Faculty of Mathematics and Physics, UniverSity of Ljubljana \& Institute of Mathematics, Physics and Mechanics, 1000 LuUbluana, Slovenia

Email address: dusan.repovs@guest.arnes.si 